\title{
Soft drink consumption is associated with increased incidence of the metabolic syndrome only in women
}

\author{
Yunjin Kang and Jihye Kim* \\ Department of Medical Nutrition, Graduate School of East-West Medical Science, Kyung Hee University, Yongin 446-701, \\ Republic of Korea \\ (Submitted 6 August 2016 - Final revision received 9 December 2016 - Accepted 4 January 2017 - First published online 7 Febraury 2017)
}

\section{Abstract}

Prospective studies on the association between soft drink consumption and incident risk of the metabolic syndrome (MetS) have not been carried out in Asians. We explored the sex-specific association between soft drink consumption and incident risk of the MetS in Korean adults during 10 years of follow-up. A total of 5797 subjects who were free of the MetS at baseline were studied. Soft drink consumption was assessed using a semi-quantitative FFQ. Time-dependent Cox proportional hazard model was used to examine hazard ratios (HR) of incidence of the MetS and its components in relation to soft drink consumption. In women, the multivariable-adjusted HR for developing the MetS was 1.8-fold higher in frequent consumers of soft drinks ( $\geq 4$ servings/week) compared with rare consumers (95\% CI 1.23, 2.64). The adjusted HR for elevated blood pressure increased by 2 -fold $(95 \%$ CI $1 \cdot 24,3 \cdot 14)$ and for hypertriacylglycerolaemia by $1 \cdot 9$-fold (95\% CI $1 \cdot 19,2 \cdot 88)$ in frequent consumers of soft drinks compared with rare consumers. However, in men, there was no association between soft drink consumption and incident risk of the MetS or its components. Frequent soft drink consumption was associated with increased risk of developing the MetS and its components only in middle-aged Korean women, suggesting sex differences for the risk of the MetS related to diet.

Key words: Soft drink consumption: Metabolic syndrome: Sex differences: Korean adults

The consumption of sugar-sweetened beverages (SSB), which generally include soft drinks, fruit drinks and sports drinks, is increasing worldwide in youth and adults ${ }^{(1-3)}$. Many clinical studies have reported the link between high consumption of SSB and increased obesity and diabetes mellitus ${ }^{(4)}$. Recent epidemiological studies have shown that SSB consumption is associated with risk of the metabolic syndrome (MetS) in adults $^{(5)}$. However, most studies on the association between soft drink consumption and the MetS have been performed in Western populations, although the prevalence of the MetS is rapidly increasing among Asian populations ${ }^{(6)}$. A few studies have been conducted in Asians ${ }^{(6,7)}$, but their cross-sectional designs could not conclude a cause-effect relationship between soft drink consumption and the risk of developing the MetS.

In Korea, dietary habits have gradually changed from a traditional diet to more a Westernised diet including meat and sweet foods such as desserts and sugary beverages ${ }^{(8)}$. Soft drink consumption is increasing in the Korean population ${ }^{(9,10)}$, although the amount is still lower than that in Western populations. The Korea National Health and Nutrition Examination Survey found that sweetened beverage consumption has doubled from 58 to $101 \mathrm{~g}$ over the past 3 years, and soft drinks are the major source of sugar intake from processed foods among Koreans ${ }^{(9)}$. Although soft drink consumption and the prevalence of the MetS are increasing among Koreans, no prospective study has been carried out on the relationship between soft drink consumption and incidence of the MetS.

Moreover, sex-specific associations between soft drink consumption and risk of incident MetS have not been examined, although sex difference has been suggested to play a role in the risk of chronic diseases including the MetS related to dietary factors ${ }^{(11)}$.

Therefore, we evaluated the sex-specific association between soft drink consumption and risk of incident MetS and its components, considering the influence of multiple lifestyle factors using data from the Korean Genome and Epidemiology Study (KoGES), which is a large community-based cohort study.

\section{Methods \\ Study subjects}

The KoGES, a large-scale, community-based cohort study, was initiated in 2001. Initially, the target population consisted of 10030 Korean adults aged 40-69 years and living in Ansan (urban) and Ansung (rural) areas. All participants responded

Abbreviations: BP, blood pressure; HR, hazard ratios; MET, metabolic equivalents; MetS, metabolic syndrome; SSB, sugar-sweetened beverages.

* Corresponding author: J. Kim, fax +82 31204 8119, email kjhye@khu.ac.kr 
to a baseline examination (2001-2002) with questionnaires including demographic information, socio-economic status, lifestyle, dietary intake, medical history, health examinations and biochemical measurements. Follow-up examinations were performed every 2 years over a 10-year period (2009-2010). The present study was approved by the Institutional Review Board of the Korea Centers for Disease Control and Prevention (KCDC). Informed written consent was obtained from all participants.

Of the original 10030 participants, participants without the MetS ( $n$ 7053) at baseline were included. We excluded participants who refused to participate in follow-up examinations ( $n$ 747), those who provided insufficient information ( $n$ 313), those who did not respond to dietary examination $(n 59)$ and those who had CVD or cancer ( $n$ 137). After exclusion, a total of 5797 participants (3027 men and 2770 women) were included in the analysis during the 10-year period. A follow-up rate of $63.3 \%$ was achieved, resulting in 33269 person-years accrued. The average follow-up period was 5.7 years (68.8 months).

\section{Dietary assessment}

Trained dietitians examined dietary intake both at baseline and at the second follow-up examination (2005-2006) using a 103-item, semi-quantitative FFQ. The FFQ was developed and validated by the $\mathrm{KCDC}^{(12)}$. In the FFQ, participants reported the frequency and portion sizes of soft drink consumption during the past year. Soft drink consumption was estimated by the questions 'How often do you consume soft drinks (carbonated beverages, e.g., Cola and Sprite)?' for frequency and 'How much soft drink do you consume at once?' for portion size. The original responses for frequency included none or rarely, once/month, two to three times per month, one to two times per week, three to four times per week, five to six times per week, one to two times per $\mathrm{d}$, three to four times per $\mathrm{d}$ and $\geq 5$ times/d. The response options for portion size were as follows: $1 / 2$ cup $(100 \mathrm{ml})$, one cup ( $200 \mathrm{ml}$ - one serving) and two or more cups $(\geq 400 \mathrm{ml})$. For analysis, the nine response items were converted to frequency per week, and the serving per week was calculated on the basis of frequency and portion size (mean frequency/week $\times 0.5$ for $1 / 2$ cup, $\times 1 \cdot 0$ for one cup, $\times 2 \cdot 0$ for two or more cups) and then categorised into four groups: none or rarely, $<1$ serving/week, $\geq 1$ serving/week to $<4$ servings/week and $\geq 4$ servings/week. Nutrient intakes were measured using a database developed by the Rural Development and Administration.

\section{Health examination}

Trained professionals conducted a comprehensive health examination under the KCDC protocol. Height and body weight were measured to the nearest $0.1 \mathrm{~cm}$ and $0.1 \mathrm{~kg}$, respectively, with no shoes and while wearing light clothing. BMI was defined as weight $(\mathrm{kg}) /$ height squared $\left(\mathrm{m}^{2}\right)$. Waist circumference (WC) measurements were repeated three times, and then averaged after measuring to the nearest $0.1 \mathrm{~cm}$ at the narrowest point between the lowest rib and the right iliac crest. Blood pressure (BP) was measured after participants had rested for more than $5 \mathrm{~min}$ in a sitting position (Baumanometer-Standby; W.A. Baum Co. Inc.) ${ }^{(13)}$. Systolic blood pressure (SBP) and diastolic blood pressure (DBP) were calculated at Korotkoff phase I and Korotkoff phase V, respectively. Averaged values of left and right arms were measured a few times at 30-s intervals. Blood samples were collected to measure fasting blood glucose (FBG), TAG and HDL-cholesterol. The blood samples were collected after at least $8 \mathrm{~h}$ of fasting at baseline and during every follow-up examination. The concentrations of glucose, TAG and HDL-cholesterol in plasma were enzymatically measured using an autoanalyzer (ADVIA 1650; Bayer HealthCare) ${ }^{(14)}$. Incidence of the MetS was diagnosed at the follow-up examination by a physician.

\section{Definition of the metabolic syndrome}

The MetS was diagnosed using criteria based on the National Cholesterol Education Program Adult Treatment Panel III definition $^{(15)}$. The MetS is defined as the presence of more than three of the following indicators: (1) abdominal obesity (WC $\geq 90 \mathrm{~cm}$ for men or $\geq 80 \mathrm{~cm}$ for women), (2) elevated BP (SBP $\geq 130 \mathrm{mmHg}$ or $\mathrm{DBP} \geq 85 \mathrm{mmHg}$ ), use of antihypertensive medication, hypertension diagnosis by a physician, (3) high blood glucose (FBG $\geq 5.6$ $\mathrm{mmol} / \mathrm{l})$, current use of insulin or oral hypoglycaemic medication, diabetes diagnosis by a physician, (4) hypertriacylglycerolaemia (TAG $\geq 1.7 \mathrm{mmol} / \mathrm{l}$ ) and (5) low HDL-cholesterol (HDL-cholesterol $<1.0 \mathrm{mmol} / \mathrm{l}$ in men or $<1.3 \mathrm{mmol} / 1$ in women).

\section{Other measurements}

Demographic characteristics, socio-economic status and lifestyle factors were examined from the baseline questionnaires. Income level was divided into four groups: $<1$ million KRW, 1-2 million KRW, 2-3 million KRW and $\geq 3$ million KRW. Education level was categorised into three groups: $\leq 6$ years (elementary school level), 7-12 years (middle/high school level) and $>12$ years (college level) of education. Smoking status was classified as follows: non-smoker, former smoker or current smoker. Alcohol intake was classified as follows: non-drinker, former drinker or current drinker. Physical activity was self-reported by a questionnaire. The questionnaire asked how many hours per day was spent performing physical activities according to its intensity (sedentary, very light, light, moderate, vigorous). Physical activities are presented as metabolic equivalents.

\section{Statistical analysis}

All the data were analysed using SAS software version 9.3 (SAS Institute). The results are expressed as percentages (categorical) or as means with their standard deviations (continuous). Differences in baseline characteristics by sex were examined by the $\chi^{2}$ test for categorical variables or Student's $t$ test for continuous variables. Differences in characteristics across soft drink consumption were evaluated by the $\chi^{2}$ tests or a generalised linear model with post hoc Tukey's honestly significant difference test.

Time-dependent Cox proportional hazard models were used as a method of survival analysis to examine the hazard ratios (HR) and $95 \%$ CI for the incident risk of the MetS and its individual components according to soft drink consumption. Survival analysis was performed separately in men and women. In multivariable adjusted models, model 1 was adjusted for age 
and model 2 was adjusted for age, income level, education level, alcohol consumption, smoking status, physical activity, BMI, energy intake, percentage of fat from energy, fibre intake and the presence of diseases (diabetes and hypertension). For the selection of variables for adjustment in the multivariable model, potential confounders from the previously published scientific literature were taken into account with the statistical approach, such as stepwise procedures or comparing adjusted and unadjusted effect estimates ${ }^{(16)}$. Individuals with hypertension or diabetes at baseline were excluded from the survival analysis to examine the risk of incident elevated BP and high blood glucose, respectively.

The proportional hazards assumption was assessed graphically using log-log plots and statistically using Schoenfeld's residuals $^{(17)}$. No violation of the proportional hazard assumption was detected. Tests for linear trends were performed on the basis of the median value of each category. All $P$ values $<0.05$ were considered statistically significant.

\section{Results}

\section{Characteristics of subjects at baseline}

Among 5797 subjects, a total of 2129 (1046 men and 1083 women) developed the MetS. Table 1 shows the baseline characteristics of subjects by the presence of the MetS. Subjects with the MetS were older, more likely to be women, to live in the rural areas, to be exercisers, to have lower income and less likely to be educated compared with subjects without the MetS. In addition, subjects with the MetS had higher intakes of energy from carbohydrate as well as lower intakes of energy from fat compared with subjects without the MetS.

\section{Characteristics of subjects according to soft drink consumption}

Table 2 shows the characteristics of subjects according to soft drink consumption. Men consuming $\geq 4$ servings/week of soft drinks were younger, more likely to have higher income, more likely to be educated and current smokers compared with rare consumers (none or rarely). Women consuming $\geq 4$ servings/ week of soft drinks were younger and more likely to have higher income, more likely to be educated and current drinkers compared with rare consumers. Regardless of sex, frequent consumers had higher intakes of energy, mostly energy from fat, along with lower intakes of energy from carbohydrates compared with rare consumers.

\section{Association between soft drink consumption and incidence} of the metabolic syndrome and its individual components

The relative risks for the MetS and its components according to soft drink consumption by sex are shown in Table 3. There was no association between soft drink consumption and risk of the MetS or its components in men. The risk of incident MetS increased significantly in women consuming $\geq 4$ servings/week of soft drinks compared with rare consumers after adjustment for potential confounding factors such as age, income,
Table 1. Characteristics of the study subjects at baseline (Numbers and percentages (categorised variables); mean values and standard deviations (continuous variables))

\begin{tabular}{|c|c|c|c|c|c|}
\hline & \multicolumn{2}{|c|}{$\begin{array}{c}\text { MetS } \\
(n 2129)\end{array}$} & \multicolumn{2}{|c|}{$\begin{array}{c}\text { Non-MetS } \\
(n \text { 3668) }\end{array}$} & \multirow[b]{2}{*}{$P$} \\
\hline & $n$ & $\%$ & $n$ & $\%$ & \\
\hline Age (years) & & & & & $<0.0001$ \\
\hline Mean & \multicolumn{2}{|c|}{$52 \cdot 6$} & \multicolumn{2}{|c|}{$50 \cdot 0$} & \\
\hline SD & \multicolumn{2}{|c|}{8.6} & \multicolumn{2}{|c|}{8.6} & \\
\hline \multicolumn{5}{|l|}{ No. of subjects (\%) } & 0.0003 \\
\hline Men & 1046 & $49 \cdot 1$ & 1981 & $54 \cdot 0$ & \\
\hline Women & 1083 & $50 \cdot 9$ & 1685 & $46 \cdot 0$ & \\
\hline \multicolumn{5}{|l|}{ Area of residence (\%) } & $<0.0001$ \\
\hline Rural area (Ansung) & 1211 & $56 \cdot 9$ & 1392 & $38 \cdot 0$ & \\
\hline Urban area (Ansan) & 918 & $43 \cdot 1$ & 2276 & $62 \cdot 1$ & \\
\hline \multicolumn{5}{|l|}{ Income level (KRW/month) (\%) } & $<0.0001$ \\
\hline$<1$ million & 791 & $37 \cdot 2$ & 959 & $26 \cdot 2$ & \\
\hline 1-2 million & 658 & 30.9 & 1121 & $30 \cdot 6$ & \\
\hline 2-3 million & 343 & $16 \cdot 1$ & 800 & $21 \cdot 8$ & \\
\hline$\geq 3$ million & 337 & $15 \cdot 8$ & 788 & 21.5 & \\
\hline \multicolumn{5}{|l|}{ Educational level (\%) } & $<0.0001$ \\
\hline Elementary school ( $\leq 6$ years) & 732 & 34.4 & 854 & $23 \cdot 3$ & \\
\hline Middle/high school ( $7-12$ years) & 1118 & 52.5 & 2215 & $60 \cdot 4$ & \\
\hline College or higher (>12 years) & 279 & $13 \cdot 1$ & 599 & $16 \cdot 3$ & \\
\hline \multicolumn{5}{|l|}{ Smoking status (\%) } & 0.1520 \\
\hline Non-smokers & 1216 & $57 \cdot 1$ & 2039 & $55 \cdot 6$ & \\
\hline Former smokers & 323 & $15 \cdot 2$ & 628 & $17 \cdot 1$ & \\
\hline Current smokers & 590 & $27 \cdot 7$ & 1001 & $27 \cdot 3$ & \\
\hline \multicolumn{5}{|l|}{ Alcohol consumption (\%) } & 0.5305 \\
\hline Non-drinkers & 914 & 42.9 & 1523 & 41.5 & \\
\hline Former drinkers & 133 & $6 \cdot 3$ & 225 & $6 \cdot 1$ & \\
\hline Current drinkers & 1082 & $50 \cdot 8$ & 1920 & $52 \cdot 3$ & \\
\hline \multicolumn{5}{|l|}{ Physical activity (MET/d) } & $<0.0001$ \\
\hline Mean & \multicolumn{2}{|c|}{$24 \cdot 4$} & \multicolumn{2}{|c|}{$22 \cdot 8$} & \\
\hline SD & \multicolumn{2}{|c|}{$15 \cdot 4$} & \multicolumn{2}{|c|}{$14 \cdot 6$} & \\
\hline \multicolumn{5}{|l|}{ Total energy intake $(\mathrm{kJ} / \mathrm{d})$} & 0.0715 \\
\hline Mean & \multicolumn{2}{|c|}{8321.9} & \multicolumn{2}{|c|}{$8175 \cdot 5$} & \\
\hline SD & \multicolumn{2}{|c|}{$3255 \cdot 1$} & 276 & $9 \cdot 8$ & \\
\hline Total energy intake (kcal/d) & & & & & \\
\hline Mean & 198 & & 195 & $4 \cdot 2$ & \\
\hline SD & 77 & & 66 & & \\
\hline Percentage from energy & & & & & \\
\hline Carbohydrate & & & & & $<0.0001$ \\
\hline Mean & 7 & & 71 & & \\
\hline SD & 7 & & 7 & & \\
\hline Protein & & & & & 0.0624 \\
\hline Mean & 15 & & 13 & & \\
\hline SD & & & 2 & & \\
\hline Fat & & & & & $<0.0001$ \\
\hline Mean & $1<$ & & 15 & & \\
\hline SD & & & 5 & & \\
\hline BMl $\left(\mathrm{kg} / \mathrm{m}^{2}\right)$ & $24 \cdot$ & $2 \cdot 8$ & $23 \cdot 1$ & 2.7 & $<0.0001$ \\
\hline
\end{tabular}

MetS, metabolic syndrome.

education, smoking status, alcohol intake, physical activity, BMI, intakes of energy, percentage of fat, fibre intake and the presence of diseases (HR 1.82; 95\% CI 1.24, 2.68, $P_{\text {for trend }}=$ 0.0005). Women consuming $\geq 4$ servings/week of soft drinks also had greater risks of elevated BP (HR 1.97; $95 \%$ CI $1 \cdot 23$, $\left.3 \cdot 14, P_{\text {for trend }}=0.0242\right)$ and hypertriacylglycerolaemia (HR 1.90; $95 \%$ CI $\left.1.22,2.96, P_{\text {for trend }}=0.0030\right)$ compared with rare consumers after adjustment for confounders.

The relative risks for the MetS and its components according to soft drink consumption and area of residence are shown in Table 4. There was no association between soft drink 
Table 2. Characteristics of the study subjects according to soft drink consumption

(Numbers and percentages (categorised variables); mean values and standard deviations (continuous variables))

\begin{tabular}{|c|c|c|c|c|c|c|c|c|c|}
\hline & \multicolumn{2}{|c|}{ Rarely or never } & \multicolumn{2}{|c|}{$<1 /$ week } & \multicolumn{2}{|c|}{$\geq 1$ to $<4 /$ week } & \multicolumn{2}{|c|}{$\geq 4 /$ week } & \multirow[b]{2}{*}{$P$} \\
\hline & $n$ & $\%$ & $n$ & $\%$ & $n$ & $\%$ & $n$ & $\%$ & \\
\hline \multicolumn{10}{|l|}{ Men } \\
\hline No. of subjects (\%) & 1060 & 35 & 1180 & 39 & 671 & 22 & 116 & 3.8 & \\
\hline Age (years) & \multirow{2}{*}{\multicolumn{2}{|c|}{$53.2^{\mathrm{a}}$}} & & & & & & & $<0.0001$ \\
\hline Mean & & & \multicolumn{2}{|c|}{$51 \cdot 2^{\mathrm{b}}$} & & & & & \\
\hline SD & & & & & & & & & \\
\hline Area of residence (\%) & & & & & & & & & 0.0020 \\
\hline Rural area (Ansung) & 496 & $46 \cdot 8$ & 548 & $46 \cdot 4$ & 257 & $38 \cdot 3$ & 55 & $47 \cdot 4$ & \\
\hline Urban area (Ansan) & 564 & $53 \cdot 2$ & 632 & $53 \cdot 6$ & 414 & 61.7 & 61 & 52.6 & \\
\hline Income level (KRW/month) (\%) & & & & & & & & & $<0.0001$ \\
\hline$<1$ million & 324 & $32 \cdot 3$ & 311 & $26 \cdot 4$ & 129 & $19 \cdot 2$ & 30 & 25.9 & \\
\hline 1-2 million & 309 & $29 \cdot 2$ & 368 & $31 \cdot 2$ & 222 & 33.1 & 32 & $27 \cdot 6$ & \\
\hline 2-3 million & 210 & $19 \cdot 8$ & 225 & $19 \cdot 1$ & 164 & 24.4 & 19 & $16 \cdot 4$ & \\
\hline$\geq 3$ million & 199 & $18 \cdot 8$ & 276 & 23.4 & 156 & 23.3 & 35 & $30 \cdot 2$ & \\
\hline Educational level (\%) & & & & & & & & & $<0.0001$ \\
\hline Elementary school ( $\leq 6$ years) & 265 & $25 \cdot 0$ & 222 & $18 \cdot 8$ & 90 & $13 \cdot 4$ & 15 & $12 \cdot 9$ & \\
\hline Middle/high school ( $7-12$ years) & 583 & $55 \cdot 0$ & 684 & $58 \cdot 0$ & 434 & 64.7 & 68 & 58.6 & \\
\hline College or higher ( $>12$ years) & 212 & $20 \cdot 0$ & 274 & $23 \cdot 2$ & 147 & 21.9 & 33 & 28.5 & \\
\hline Smoking status (\%) & & & & & & & & & 0.0017 \\
\hline Non-smokers & 189 & $17 \cdot 8$ & 277 & 23.5 & 122 & $18 \cdot 2$ & 18 & 15.5 & \\
\hline Former smokers & 328 & 30.9 & 368 & $31 \cdot 2$ & 194 & 28.9 & 31 & $26 \cdot 7$ & \\
\hline Current smokers & 543 & $51 \cdot 2$ & 535 & $45 \cdot 3$ & 355 & 52.9 & 67 & 57.8 & \\
\hline Alcohol consumption (\%) & & & & & & & & & 0.5757 \\
\hline Non-drinkers & 183 & $17 \cdot 3$ & 235 & 19.9 & 133 & $19 \cdot 8$ & 21 & $18 \cdot 1$ & \\
\hline Former drinkers & 109 & $10 \cdot 3$ & 103 & 8.7 & 68 & $10 \cdot 1$ & 13 & $11 \cdot 2$ & \\
\hline Current drinkers & 768 & 72.5 & 842 & 71.4 & 470 & $70 \cdot 0$ & 82 & $70 \cdot 7$ & \\
\hline Physical activity (MET/d) & & & & & & & & & 0.3516 \\
\hline Mean & & & & & & & & & \\
\hline SD & & & & & & & & & \\
\hline Total energy intake $(\mathrm{kJ} / \mathrm{d})$ & & & & & & & & & $<0.0001$ \\
\hline Mean & & & & & & & & & \\
\hline SD & & & & & & & & & \\
\hline Total energy intake (kcal/d) & & & & & & & & & \\
\hline Mean & & & & & & & & & \\
\hline SD & & & & & & & & & \\
\hline Percentage from energy & & & & & & & & & \\
\hline Carbohydrate (\%) & & & & & & & & & $<0.0001$ \\
\hline Mean & & & & & & & & & \\
\hline SD & & & & & & & & & \\
\hline Protein (\%) & & & & & & & & & $<0.0001$ \\
\hline Mean & & & & & & & & & \\
\hline SD & & & & & & & & & \\
\hline Fat $(\%)$ & & & & & & & & & $<0.0001$ \\
\hline Mean & & & & & & & & & \\
\hline SD & & & & & & & & & \\
\hline BMI $\left(\mathrm{kg} / \mathrm{m}^{2}\right)$ & & & & & & & & & 0.0095 \\
\hline Mean & & & & & & & & & \\
\hline SD & & & & & & & & & \\
\hline Components of the metabolic syndrome & & & & & & & & & \\
\hline Waist circumference $(\mathrm{cm})$ & & & & & & & & & 0.1260 \\
\hline Mean & & & & & & & & & \\
\hline SD & & & & & & & & & \\
\hline Systolic blood pressure $(\mathrm{mmHg})$ & & & & & & & & & 0.0086 \\
\hline Mean & & & & & & & & & \\
\hline SD & & & & & & & & & \\
\hline Diastolic blood pressure $(\mathrm{mmHg})$ & & & & & & & & & 0.1713 \\
\hline Mean & & & & & & & & & \\
\hline SD & & & & & & & & & \\
\hline Fasting blood glucose $(\mathrm{mmol} / \mathrm{l})$ & & & & & & & & & 0.0150 \\
\hline Mean & & & & & & & & & \\
\hline SD & & & & & & & & & \\
\hline TAG (mmol/l) & & & & & & & & & 0.4696 \\
\hline Mean & & & & & & & & & \\
\hline SD & & & & & & & & & \\
\hline HDL-cholesterol (mmol/l) & & & & & & & & & 0.1198 \\
\hline Mean & & & & & & & & & \\
\hline SD & & & & & & & & & \\
\hline Women & & & & & & & & & \\
\hline No. of subjects (\%) & 1404 & 51 & 996 & 36 & 315 & 11 & 55 & 2 & \\
\hline Age (years) & & & & & & & & & $<0.0001$ \\
\hline Mean & & & & & & & & & \\
\hline SD & & & & & & & & & \\
\hline
\end{tabular}


Table 2. Continued

\begin{tabular}{|c|c|c|c|c|c|c|c|c|c|}
\hline & \multicolumn{2}{|c|}{ Rarely or never } & \multicolumn{2}{|c|}{$<1 /$ week } & \multicolumn{2}{|c|}{$\geq 1$ to $<4 /$ week } & \multicolumn{2}{|c|}{$\geq 4 /$ week } & \multirow[b]{2}{*}{$P$} \\
\hline & $n$ & $\%$ & $n$ & $\%$ & $n$ & $\%$ & $n$ & $\%$ & \\
\hline \multicolumn{9}{|l|}{ Area of residence (\%) } & \multirow[t]{3}{*}{0.0001} \\
\hline Rural area (Ansung) & 613 & 43.7 & 479 & $48 \cdot 1$ & 119 & 37.8 & 36 & 65.5 & \\
\hline Urban area (Ansan) & 791 & $56 \cdot 3$ & 517 & 51.9 & 196 & 62.2 & 19 & 34.6 & \\
\hline \multicolumn{9}{|l|}{ Income level (\%) } & \multirow{5}{*}{0.0050} \\
\hline$<1$ million & 499 & 35.5 & 337 & 33.8 & 80 & $25 \cdot 4$ & 22 & $40 \cdot 0$ & \\
\hline 1-2 million & 432 & 30.8 & 306 & 30.7 & 93 & 29.5 & 17 & 30.9 & \\
\hline 2-3 million & 251 & $17 \cdot 9$ & 190 & $19 \cdot 1$ & 80 & $25 \cdot 4$ & 4 & $7 \cdot 3$ & \\
\hline$\geq 3$ million & 222 & $15 \cdot 8$ & 163 & $16 \cdot 4$ & 62 & $19 \cdot 7$ & 12 & 21.8 & \\
\hline \multicolumn{9}{|l|}{ Educational level (KRW/month) (\%) } & \multirow{4}{*}{0.0175} \\
\hline Elementary school ( $\leq 6$ years) & 531 & 37.8 & 361 & $36 \cdot 2$ & 84 & $26 \cdot 7$ & 18 & $32 \cdot 7$ & \\
\hline Middle/high school (7-12 years) & 771 & 54.9 & 555 & $55 \cdot 7$ & 207 & $65 \cdot 7$ & 31 & $56 \cdot 4$ & \\
\hline College or higher ( $>12$ years) & 102 & $7 \cdot 3$ & 80 & 8.0 & 24 & 7.6 & 6 & 10.9 & \\
\hline \multicolumn{10}{|l|}{ Smoking status (\%) } \\
\hline Non-smokers & 1332 & 94.9 & 964 & $96 \cdot 8$ & 301 & 95.6 & 52 & 94.6 & \multirow[t]{3}{*}{0.1932} \\
\hline Former smokers & 15 & 1.1 & 10 & 1.0 & 5 & 1.6 & 0 & 0.0 & \\
\hline Current smokers & 57 & $4 \cdot 1$ & 22 & $2 \cdot 2$ & 9 & 2.9 & 3 & 5.5 & \\
\hline Alcohol consumption (\%) & & & & & & & & & $<0.0001$ \\
\hline Non-drinkers & 992 & 70.7 & 671 & 67.4 & 173 & 54.9 & 29 & 52.7 & \\
\hline Former drinkers & 36 & $2 \cdot 6$ & 17 & 1.7 & 11 & 3.5 & 1 & 1.8 & \\
\hline Current drinkers & 376 & $26 \cdot 8$ & 308 & $30 \cdot 9$ & 131 & 41.6 & 25 & 45.5 & \\
\hline Physical activity (MET/d) & & & & & & & & & 0.0009 \\
\hline Mean & & & & & & & & & \\
\hline $\mathrm{SD}$ & & & & & & & & & \\
\hline Total energy intake $(\mathrm{kJ} / \mathrm{d})$ & & & & & & & & & $<0.0001$ \\
\hline Mean & & & & & & & & 8.5 & \\
\hline SD & & & & & & & & & \\
\hline Total energy intake $(\mathrm{kcal} / \mathrm{d})$ & & & & & & & & & \\
\hline Mean & & & & & & & & & \\
\hline SD & & & & & & & & & \\
\hline Percentage from energy & & & & & & & & & \\
\hline Carbohydrate (\%) & & & & & & & & & $<0.0001$ \\
\hline Mean & & & & & & & & & -0.000 \\
\hline SD & & & & & & & & & \\
\hline Protein (\%) & & & & & & & & & $<0.0001$ \\
\hline Mean & & & & & & & & & \\
\hline SD & & & & & & & & & \\
\hline Fat $(\%)$ & & & & & & & & & $<0.0001$ \\
\hline Mean & & & & & & & & & \\
\hline SD & & & & & & & & & \\
\hline BMI $\left(\mathrm{kg} / \mathrm{m}^{2}\right)$ & & & & & & & & & 0.0175 \\
\hline Mean & & & & & & & & & \\
\hline SD & & & & & & & & & \\
\hline Component of the metabolic syndrome & & & & & & & & & \\
\hline Waist circumference $(\mathrm{cm})$ & & & & & & & & & 0.1359 \\
\hline $\begin{array}{l}\text { Mean } \\
\text { SD }\end{array}$ & & & & & & & & & \\
\hline Systolic blood pressure $(\mathrm{mmHg})$ & & & & & & & & & 0.0546 \\
\hline Mean & & & & & & & & & \\
\hline SD & & & & & & & & & \\
\hline Diastolic blood pressure $(\mathrm{mmHg})$ & & & & & & & & & 0.1413 \\
\hline Mean & & & & & & & & & \\
\hline SD & & & & & & & & & \\
\hline Fasting blood glucose $(\mathrm{mmol} / \mathrm{l})$ & & & & & & & & & 0.1088 \\
\hline Mean & & & & & & & & & \\
\hline SD & & & & & & & & & \\
\hline TAG (mmol/l) & & & & & & & & & 0.4205 \\
\hline Mean & & & & & & & & & \\
\hline SD & & & & & & & & & \\
\hline HDL-cholesterol (mmol/l) & & & & & & & & & 0.5610 \\
\hline Mean & & & & & & & & & \\
\hline SD & & & & & & & & & \\
\hline
\end{tabular}

a,b,c Multiple comparisons are given by post hoc Tukey's HSD test $(P<0.05)$.

consumption and risk of the MetS or its components either in the rural area or in the urban area in men (data not shown). In addition, in women living in the rural area, there was no association between soft drink consumption and risk of the MetS and its components. However, in women living in the urban area, the risk of incident MetS increased significantly with consuming $\geq 4$ servings/week of soft drinks compared with rare consumers after adjustment for potential confounding factors such as age, income, education, smoking status, alcohol intake, physical activity, BMI, intakes of energy, percentage of fat, fibre intake and the presence of diseases (HR 1.71; 95\% CI 0.84, $3 \cdot 47, P_{\text {for trend }}=0 \cdot 0021$ ). Women living in the urban area and 
Table 3. Incidence of the metabolic syndrome (MetS) and its components according to soft drink consumption by sex* (Hazard ratios (HR) and $95 \%$ confidence intervals)

\begin{tabular}{|c|c|c|c|c|c|c|c|c|c|}
\hline & \multirow{2}{*}{$\frac{\text { Rarely or never }}{\mathrm{HR}}$} & \multicolumn{2}{|c|}{$<1 /$ week } & \multicolumn{2}{|c|}{$\geq 1 /$ week to $<4 /$ week } & \multicolumn{2}{|c|}{$\geq 4 /$ week } & \multirow[b]{2}{*}{$P_{\text {for trend }}$} & \multirow[b]{2}{*}{$P_{\text {for interaction }}$} \\
\hline & & $\mathrm{HR}$ & $95 \% \mathrm{Cl}$ & $\mathrm{HR}$ & $95 \% \mathrm{Cl}$ & $\mathrm{HR}$ & $95 \% \mathrm{Cl}$ & & \\
\hline \multicolumn{10}{|l|}{ Total } \\
\hline MetS & 1.00 & 0.95 & $0.87,1.05$ & $1 \cdot 18$ & $1.04,1.34$ & 1.35 & $1.06,1 \cdot 71$ & 0.5123 & 0.1041 \\
\hline Abdominal obesity & 1.00 & 0.91 & $0.81,1.02$ & 1.11 & $0.96,1.29$ & $1 \cdot 17$ & $0.86,1.60$ & 0.3434 & 0.5079 \\
\hline Elevated blood pressure $\dagger$ & 1.00 & 0.93 & $0.83,1.04$ & 1.28 & $1.12,1.48$ & 1.55 & $1 \cdot 18,2.03$ & 0.0414 & 0.3769 \\
\hline High fasting blood glucoseł & 1.00 & 0.86 & $0.78,0.95$ & 1.09 & $0.96,1.24$ & $1 \cdot 20$ & $0.94,1.53$ & 0.8967 & 0.8193 \\
\hline High TAG & 1.00 & 0.89 & $0.80,0.98$ & $1 \cdot 26$ & $1 \cdot 10,1.43$ & $1 \cdot 20$ & $0.91,1.60$ & 0.8682 & 0.5107 \\
\hline Low HDL-cholesterol & 1.00 & 0.90 & $0.83,0.98$ & 1.05 & $0.94,1.17$ & $1 \cdot 17$ & $0.96,1.44$ & 0.9013 & 0.8797 \\
\hline \multicolumn{10}{|l|}{ Men } \\
\hline \multicolumn{10}{|l|}{ MetS } \\
\hline$n$ & 1060 & & 1180 & & 371 & & 116 & & \\
\hline No. of cases & 385 & & 382 & & 234 & & 45 & & \\
\hline Model 1 & 1.00 & 0.81 & $0.70,0.93$ & 0.99 & $0.84,1 \cdot 17$ & 1.08 & $0.79,1.47$ & 0.9808 & \\
\hline Model 2 & 1.00 & 0.86 & $0.74,0.99$ & 0.98 & $0.83,1.16$ & 1.09 & $0.79,1.49$ & 0.9531 & \\
\hline \multicolumn{10}{|l|}{ Abdominal obesity } \\
\hline$n$ & 1127 & & 1237 & & 565 & & 109 & & \\
\hline No. of cases & 278 & & 273 & & 167 & & 28 & & \\
\hline Model 1 & 1.00 & 0.84 & $0.71,0.99$ & 1.08 & $0.89,1.31$ & $1 \cdot 11$ & $0.75,1.65$ & 0.5280 & \\
\hline Model 2 & 1.00 & 0.87 & $0.73,1.03$ & 1.07 & $0.87,1.31$ & $1 \cdot 11$ & $0.74,1.65$ & 0.6012 & \\
\hline \multicolumn{10}{|l|}{ Elevated blood pressure } \\
\hline$n$ & 710 & & 813 & & 510 & & 89 & & \\
\hline No. of cases & 268 & & 311 & & 204 & & 40 & & \\
\hline Model 1† & 1.00 & 0.97 & $0.82,1 \cdot 14$ & $1 \cdot 22$ & $1.01,1.47$ & $1 \cdot 36$ & $0.98,1.90$ & 0.0159 & \\
\hline Model $2 \dagger$ & 1.00 & 0.98 & $0.83,1 \cdot 16$ & 1.22 & $1.01,1.48$ & $1 \cdot 37$ & $0.98,1.93$ & 0.0175 & \\
\hline High fasting blood glucose & & & & & & & & & \\
\hline$n$ & 1042 & & 1223 & & 678 & & 125 & & \\
\hline No. of cases & 416 & & 443 & & 264 & & 58 & & \\
\hline Model $1 \ddagger$ & 1.00 & 0.80 & $0.70,0.91$ & 0.97 & $0.83,1.14$ & $1 \cdot 20$ & $0.91,1.59$ & 0.7706 & \\
\hline Model $2 \ddagger$ & 1.00 & 0.80 & $0.70,0.92$ & 0.97 & $0.82,1.13$ & $1 \cdot 12$ & $0.85,1.49$ & 0.9527 & \\
\hline High TAG & & & & & & & & & \\
\hline$n$ & 767 & & 849 & & 511 & & 88 & & \\
\hline No. of cases & 298 & & 292 & & 223 & & 33 & & \\
\hline Model 1 & 1.00 & 0.76 & $0.64,0.89$ & $1 \cdot 10$ & $0.92,1.31$ & 0.84 & $0.58,1.20$ & 0.8361 & \\
\hline Model 2 & 1.00 & 0.78 & $0.66,0.92$ & $1 \cdot 11$ & $0.93,1.33$ & 0.90 & $0.62,1.30$ & 0.6592 & \\
\hline Low HDL-cholesterol & & & & & & & & & \\
\hline$n$ & 1092 & & 1127 & & 658 & & 123 & & \\
\hline No. of cases & 541 & & 540 & & 327 & & 66 & & \\
\hline Model 1 & 1.00 & 0.88 & $0.78,0.99$ & 1.03 & $0.89,1.18$ & $1 \cdot 13$ & $0.87,1.46$ & 0.5754 & \\
\hline Model 2 & 1.00 & 0.88 & $0.78,0.99$ & 1.02 & $0.89,1.18$ & $1 \cdot 14$ & $0.87,1 \cdot 18$ & 0.6259 & \\
\hline Women & & & & & & & & & \\
\hline MetS & & & & & & & & & \\
\hline$n$ & 1404 & & 996 & & 315 & & 55 & & \\
\hline No. of cases & 531 & & 386 & & 138 & & 28 & & \\
\hline Model 1 & 1.00 & 1.04 & $0.91,1.18$ & 1.40 & $1.16,1.69$ & 2.07 & $1.42,3.03$ & $<0.0001$ & \\
\hline Model 2 & 1.00 & 1.01 & $0.89,1.16$ & 1.39 & $1.15,1.69$ & 1.82 & $1 \cdot 24,2.68$ & 0.0005 & \\
\hline Abdominal obesity & & & & & & & & & \\
\hline$n$ & 993 & & 646 & & 206 & & 29 & & \\
\hline No. of cases & 405 & & 254 & & 82 & & 15 & & \\
\hline Model 1 & 1.00 & 0.96 & $0.82,1.12$ & $1 \cdot 11$ & $0.87,1.41$ & 1.78 & $1.06,2.99$ & 0.2532 & \\
\hline Model 2 & 1.00 & 0.95 & $0.81,1 \cdot 11$ & $1 \cdot 12$ & $0.88,1.43$ & $1 \cdot 32$ & $0.78,2.23$ & 0.4387 & \\
\hline Elevated blood pressure & & & & & & & & & \\
\hline$n$ & 1223 & & 898 & & 303 & & 45 & & \\
\hline No. of cases & 382 & & 253 & & 112 & & 19 & & \\
\hline Model $1 \dagger$ & 1.00 & 0.90 & $0.77,1.06$ & 1.37 & $1 \cdot 11,1 \cdot 70$ & $2 \cdot 24$ & $1.41,3.56$ & 0.0047 & \\
\hline Model 2† & 1.00 & 0.88 & $0.75,1.04$ & 1.32 & $1.07,1.64$ & 1.97 & $1 \cdot 23,3 \cdot 14$ & 0.0242 & \\
\hline High fasting blood glucose & & & & & & & & & \\
\hline$n$ & 1809 & & 1319 & & 407 & & 57 & & \\
\hline No. of cases & 458 & & 317 & & 120 & & 16 & & \\
\hline Model $1 \ddagger$ & 1.00 & 0.93 & $0.80,1.07$ & 1.33 & $1.08,1.62$ & 1.37 & $0.83,2.26$ & 0.0584 & \\
\hline Model $2 \ddagger$ & 1.00 & 0.90 & $0.78,1.04$ & 1.23 & $1.00,1.51$ & $1 \cdot 13$ & $0.68,1.86$ & 0.3602 & \\
\hline High TAG & & & & & & & & & \\
\hline$n$ & 1484 & & 1063 & & 331 & & 51 & & \\
\hline No. of cases & 483 & & 342 & & 122 & & 21 & & \\
\hline Model 1 & 1.00 & 0.96 & $0.84,1.11$ & 1.33 & $1.09,1.63$ & 1.74 & $1.12,2.69$ & 0.0096 & \\
\hline Model 2 & 1.00 & 0.97 & $0.84,1 \cdot 11$ & 1.40 & $1.14,1.72$ & 1.90 & $1 \cdot 22,2.96$ & 0.0030 & \\
\hline
\end{tabular}


Table 3. Continued

\begin{tabular}{|c|c|c|c|c|c|c|c|c|c|}
\hline & \multirow{2}{*}{$\frac{\text { Rarely or never }}{\text { HR }}$} & \multicolumn{2}{|c|}{$<1 /$ week } & \multicolumn{2}{|c|}{$\geq 1 /$ week to $<4 /$ week } & \multicolumn{2}{|c|}{$\geq 4 /$ week } & \multirow[b]{2}{*}{$P_{\text {for trend }}$} & \multirow[b]{2}{*}{$P_{\text {for interaction }}$} \\
\hline & & $\mathrm{HR}$ & $95 \% \mathrm{Cl}$ & $\mathrm{HR}$ & $95 \% \mathrm{Cl}$ & $\mathrm{HR}$ & $95 \% \mathrm{Cl}$ & & \\
\hline \multicolumn{10}{|c|}{ Low HDL-cholesterol } \\
\hline$n$ & 1120 & & 672 & & 261 & & 58 & & \\
\hline No. of cases & 772 & & 456 & & 172 & & 39 & & \\
\hline Model 1 & 1.00 & 0.91 & $0.81,1.02$ & 1.02 & $0.87,1.21$ & 1.21 & $0.88,1.67$ & 0.8877 & \\
\hline Model 2 & 1.00 & 0.92 & $0.82,1.03$ & 1.08 & $0.91,1.28$ & 1.27 & $0.92,1.28$ & 0.4711 & \\
\hline
\end{tabular}

* Model 1 was adjusted for age. Model 2 was adjusted for age, income level, education level, alcohol consumption, smoking status, physical activity, BMI, energy intake, percentage of fat, fibre intake and the presence of diseases.

$\dagger$ Excluded those who have hypertension at baseline from the analysis.

$\ddagger$ Excluded those who have diabetes mellitus at baseline from the analysis.

consuming $\geq 4$ servings/week of soft drinks also had greater risks of hypertriacylglycerolaemia (HR 1.76; $95 \%$ CI 0.83, 3.74, $\left.P_{\text {for trend }}=0.0004\right)$ compared with rare consumers after adjustment for confounders.

\section{Discussion}

We found that soft drink consumption was associated with a higher risk of the MetS only in women. In women, frequent consumption of soft drinks ( $\geq 4$ servings/week) increased the risk of incident MetS by $80 \%$ compared with rare consumers after adjustment for potential confounders. Frequent consumption of soft drinks also significantly increased the risks of incident elevated BP and hypertriacylglycerolaemia in women. In particular, a strong association between soft drink consumption and risk of the MetS or hypertriacylglycerolaemia was shown in women living in the urban area. However, in men, no association was found between soft drink consumption and risk of incident MetS or its components regardless of area of residence. These findings suggest sex differences in the associations between dietary factors and metabolic risks.

Our results are consistent with previous findings. In the Framingham Heart study, the incidence of the MetS was $44 \%$ higher among middle-aged adults who consumed $\geq 1$ soft drink/d compared with those who consumed $<1$ soft drink/d during 4 years of follow-up. In addition, frequent consumers of soft drinks had $25-32 \%$ higher risk of incidence of abdominal obesity, impaired fasting glucose, hypertriacylglycerolaemia and low HDL-cholesterol compared with infrequent consumers ${ }^{(18)}$. Among young university graduates, participants in the highest quintile of SSB consumption had a 2-fold higher risk of developing the MetS compared with those in the lowest quintile during 6 years of follow-up. Increased intake of SSB was also associated with a greater risk of high BP, obesity and hypertriacylglycerolaemia ${ }^{(5)}$.

Several mechanisms can explain the higher risk of the MetS associated with greater consumption of soft drinks. First, high consumption of added nutritive sweeteners such as highfructose maize syrup (the primary sweetener in soft drinks) may be associated with metabolic traits. High-fructose maize syrup used in beverages contains about $55 \%$ fructose. A human study showed that fructose over-feeding for $6 \mathrm{~d}$ led to stimulation of hepatic de novo lipogenesis and to a substantial increase in plasma TAG in young subjects ${ }^{(19)}$. High fructose intake in the form of added sugars ( $\geq 74 \mathrm{~g} / \mathrm{d})$ was associated with a $26-77 \%$ higher risk of elevated BP in US adults ${ }^{(20)}$.

The mechanism by which fructose causes elevated BP or hyperlipidaemia is not fully understood, but some possibilities have been suggested. Increased sympathetic nervous system activity, possibly triggered by insulin resistance, could lead to an increase in $\mathrm{BP}^{(21,22)}$. Another possibility includes increased activity of the renin-angiotensin system. A rat study showed that BP and TAG levels were significantly greater in fructose-fed rats than in control rats. The level of angiotensin II type 1 receptor mRNA was significantly higher in adipose tissue from fructose-fed rats than in tissue from control rats ${ }^{(23)}$.

Second, dietary habits and lifestyle behaviours among individuals consuming soft drinks might be associated with the risk of the MetS. Frequent consumers of soft drinks had dietary habits characterised by greater intakes of energy content and fat with low intakes of dietary fibre, as well as unhealthy lifestyles including smoking and alcohol consumption. Although these dietary and lifestyle factors are adjusted for in the analysis, other factors not adjusted for in the present analysis, such as dietary pattern, might influence the incidence of the MetS and its individual components. Data from the National Health and Nutrition Examination Survey showed that adults who ate more snacks, high-fat foods and fast food had a higher possibility of drinking energetically sweetened beverages such as soda, fruit drinks and coffee ${ }^{(24)}$.

Of particular interest, soft drink consumption was positively associated with the incident risk of the MetS, hypertriacylglycerolaemia and elevated BP in women only. Similarly, frequent consumption of soft drinks was related to a higher prevalence of the MetS, elevated BP and hypertriacylglycerolaemia in women only ${ }^{(6)}$. Sex might be a factor in determining the degree of association with the MetS or its components, particularly with hypertension and dyslipidaemia ${ }^{(25)}$. The sex difference may be associated with sex hormones ${ }^{(26)}$. Sex hormones such as oestrogen might participate in the activation pathway of protein kinase $\mathrm{C}$, which might influence vascular smooth muscle contraction or relaxation by increasing nitric oxide release ${ }^{(27)}$. Oestrogen also affects the renin-angiotensin system, which might be regulated differently in men and women, with endogenous oestrogen suppressing angiotensin receptor type 1 expression and angiotensinogen synthesis ${ }^{(28)}$. Besides, oestrogen enhances fat transport and increases the levels of TAG and lipoprotein in the blood, whereas androgen has the opposite effect of oestrogen ${ }^{(29)}$. Therefore, lipid levels 
Table 4. Incidence of the metabolic syndrome (MetS) and its components according to soft drink consumption by area of residence in women* (Hazard ratios (HR) and $95 \%$ confidence intervals)

\begin{tabular}{|c|c|c|c|c|c|c|c|c|}
\hline & \multirow{2}{*}{$\begin{array}{c}\text { Rarely or never } \\
\text { HR }\end{array}$} & \multicolumn{2}{|c|}{$<1 /$ week } & \multicolumn{2}{|c|}{$\geq 1 /$ week to $<4 /$ week } & \multicolumn{2}{|c|}{$\geq 4 /$ week } & \multirow[b]{2}{*}{$P_{\text {for trend }}$} \\
\hline & & $\mathrm{HR}$ & $95 \% \mathrm{Cl}$ & $\mathrm{HR}$ & $95 \% \mathrm{Cl}$ & HR & $95 \% \mathrm{Cl}$ & \\
\hline \multicolumn{9}{|c|}{ Rural area (Ansung) } \\
\hline \multicolumn{9}{|l|}{ MetS } \\
\hline$n$ & 613 & & 479 & & 19 & & 36 & \\
\hline No. of cases & 333 & & 249 & & 0 & & 20 & \\
\hline Model 1 & 1.00 & 1.22 & $1.06,1.40$ & 1.52 & $1.19,1.94$ & 1.97 & $1.26,3.08$ & 0.1778 \\
\hline Model 2 & 1.00 & 1.05 & $0.90,1.23$ & $1 \cdot 26$ & $0.98,1.63$ & 1.79 & $1 \cdot 14,2 \cdot 82$ & 0.2234 \\
\hline \multicolumn{9}{|c|}{ Abdominal obesity } \\
\hline$n$ & 333 & & 227 & & 51 & & 14 & \\
\hline No. of cases & 217 & & 129 & & 34 & & 10 & \\
\hline Model 1 & 1.00 & 1.61 & $1.33,1.95$ & $2 \cdot 26$ & $1.60,3.19$ & $2 \cdot 51$ & $1.34,4.70$ & 0.6594 \\
\hline Model 2 & 1.00 & 1.40 & $1.13,1.74$ & 1.88 & $1 \cdot 31,2.69$ & $2 \cdot 27$ & $1 \cdot 20,4 \cdot 29$ & 0.3007 \\
\hline \multicolumn{9}{|c|}{ Elevated blood pressure } \\
\hline$n$ & 488 & & 424 & & 19 & & 28 & \\
\hline No. of cases & 229 & & 170 & & 2 & & 15 & \\
\hline Model 1† & 1.00 & 0.78 & $0.66,0.93$ & $1 \cdot 10$ & $0.85,1.43$ & 1.76 & $1.05,2.94$ & 0.3181 \\
\hline Model $2 \dagger$ & 1.00 & 1.03 & $0.89,1.20$ & 1.24 & $0.98,1.57$ & 1.59 & $1 \cdot 07,2 \cdot 36$ & 0.8503 \\
\hline \multicolumn{9}{|c|}{ High fasting blood glucose } \\
\hline$n$ & 892 & & 732 & & 87 & & 39 & \\
\hline No. of cases & 293 & & 204 & & 77 & & 11 & \\
\hline Model $1 \ddagger$ & 1.00 & 0.62 & $0.53,0.72$ & 0.86 & $0.67,1.10$ & 0.77 & $0.43,1.40$ & 0.6593 \\
\hline Model $2 \ddagger$ & 1.00 & 0.73 & $0.61,0.86$ & 0.97 & $0.75,1.25$ & 0.85 & $0.47,1.55$ & 0.4517 \\
\hline \multicolumn{9}{|l|}{ High TAG } \\
\hline$n$ & 726 & & 582 & & 42 & & 34 & \\
\hline No. of cases & 267 & & 196 & & 51 & & 14 & \\
\hline Model 1 & 1.00 & 0.84 & $0.71,0.98$ & 0.97 & $0.73,1.29$ & 1.32 & $0.77,2.24$ & 0.7799 \\
\hline Model 2 & 1.00 & 0.90 & $0.76,1.07$ & 1.08 & $0.80,1.46$ & 1.56 & $0.91,2.68$ & 0.3870 \\
\hline \multicolumn{9}{|c|}{ Low HDL-cholesterol } \\
\hline$n$ & 557 & & 347 & & 07 & & 36 & \\
\hline No. of cases & 426 & & 247 & & 30 & & 26 & \\
\hline Model 1 & 1.00 & 1.32 & $1 \cdot 15,1.51$ & 1.57 & $1.25,1.97$ & 1.77 & $1 \cdot 20,2 \cdot 62$ & 0.6176 \\
\hline Model 2 & 1.00 & 1.03 & $0.89,1.20$ & $1 \cdot 24$ & $0.98,1.57$ & 1.59 & $1 \cdot 07,2 \cdot 36$ & 0.8564 \\
\hline \multicolumn{9}{|c|}{ Urban area (Ansan) } \\
\hline MetS & & & & & & & & \\
\hline$n$ & 791 & & 517 & & 96 & & 19 & \\
\hline No. of cases & 198 & & 137 & & 8 & & 8 & \\
\hline Model 1 & 1.00 & 0.88 & $0.73,1.05$ & 1.34 & $1.04,1.72$ & 1.73 & $0.86,2.48$ & $<0.0001$ \\
\hline Model 2 & 1.00 & 1.10 & $0.90,1.35$ & 1.61 & $1 \cdot 24,2 \cdot 10$ & 1.71 & $0.84,3.47$ & 0.0021 \\
\hline Abdominal obes & & & & & & & & \\
\hline$n$ & 660 & & 419 & & 55 & & 15 & \\
\hline No. of cases & 188 & & 125 & & 8 & & 5 & \\
\hline Model 1 & 1.00 & 1.50 & $1.23,1.82$ & 1.72 & $1 \cdot 28,2 \cdot 32$ & 2.09 & $0.87,5.04$ & 0.0749 \\
\hline Model 2 & 1.00 & 1.54 & $1.24,1.92$ & 1.77 & $1 \cdot 29,2.42$ & 1.44 & $0.59,3.51$ & 0.3242 \\
\hline Elevated blood & & & & & & & & \\
\hline$n$ & 735 & & 474 & & 84 & & 17 & \\
\hline No. of cases & 153 & & 83 & & 50 & & 4 & \\
\hline Model 1† & 1.00 & 0.59 & $0.46,0.74$ & 1.05 & $0.79,1.41$ & 1.00 & $0.37,2.67$ & 0.0345 \\
\hline Model $2 \dagger$ & 1.00 & 1.11 & $0.95,1.31$ & 1.29 & $1.03,1.61$ & 1.38 & $0.80,2.40$ & 0.4012 \\
\hline High fasting blo & & & & & & & & \\
\hline$n$ & 917 & & 587 & & 20 & & 18 & \\
\hline No. of cases & 165 & & 113 & & 53 & & 5 & \\
\hline Model $1 \ddagger$ & 1.00 & 0.61 & $0.50,0.75$ & 0.87 & $0.66,1.15$ & 0.91 & $0.38,2.19$ & 0.0024 \\
\hline Model $2 \ddagger$ & 1.00 & 0.82 & $0.66,1.01$ & 1.05 & $0.78,1.40$ & 1.09 & $0.45,2.65$ & 0.0232 \\
\hline High TAG & & & & & & & & \\
\hline$n$ & 758 & & 481 & & 89 & & 17 & \\
\hline No. of cases & 216 & & 146 & & 1 & & 7 & \\
\hline Model 1 & 1.00 & 0.80 & $0.67,0.95$ & 1.17 & $0.92,1.50$ & 1.41 & $0.67,2.97$ & 0.0005 \\
\hline Model 2 & 1.00 & 0.95 & $0.78,1.15$ & 1.38 & $1.06,1.78$ & 1.76 & $0.83,3.73$ & 0.0004 \\
\hline Low HDL-chole & & & & & & & & \\
\hline$n$ & 563 & & 325 & & 54 & & 22 & \\
\hline No. of cases & 346 & & 209 & & 2 & & 13 & \\
\hline Model 1 & 1.00 & 1.29 & $1.11,1.49$ & 1.42 & $1.14,1.76$ & 1.66 & $0.96,2.87$ & 0.6799 \\
\hline Model 2 & 1.00 & 1.11 & $0.95,1.31$ & 1.29 & $1.03,1.61$ & $1 \cdot 38$ & $0.80,2.40$ & 0.4094 \\
\hline
\end{tabular}

* Model 1 was adjusted for age. Model 2 was adjusted for age, income level, education level, alcohol consumption, smoking status, physical activity, BMI, energy intake, \% fat, fibre intake and the presence of diseases.

$\dagger$ Excluded those who have hypertension at baseline from the analysis.

$\ddagger$ Excluded those who have diabetes mellitus at baseline from the analysis. 
could be differently regulated between men and women. The levels of TAG and lipoproteins appear to be more sensitive to perturbations in dietary carbohydrates or fats in women than in men. Li et al. reported that a low-fat, high-carbohydrate diet for 6 weeks $v$. an average American diet increased TAG levels only in women ${ }^{(30)}$. In the present study, changes in lipid metabolism caused by sex hormones might have contributed to a greater association between soft drink consumption and hypertriacylglycerolaemia/MetS in women.

A positive association between soft drink consumption and risk of the MetS or hypertriacylglycerolaemia was stronger in women living in the urban area than those living in the rural area. The urban/rural differences may be due to the differences in dietary habits, physical activity and education of subjects ${ }^{(31)}$. If women living in the urban area had a healthy, balanced diet and a healthy lifestyle compared with those living in the rural area, frequent consumption of soft drinks as an unhealthy dietary habit would have had a greater impact on the MetS/hypertriacylglycerolaemia in women living in the urban area.

To the best of our knowledge, this is the first prospective study to investigate associations between soft drink consumption and incident MetS or its components in an Asian population. The study identified sex differences in the association between soft drink intake and risk of the MetS using data from a large-scale, cohort study, and the analysis considered multiple confounders including lifestyle factors and nutrient intakes. Despite these strengths, this study has some limitations. Confounding factors such as lifestyle or dietary patterns that were not considered in the analysis might affect the metabolic risks associated with soft drink consumption. The present study did not examine the consumption of diet soft drinks, which might have affected the metabolic risks. Our findings cannot be generalised to other age groups because the cohort comprised middle-aged Korean adults.

In conclusion, frequent soft drink consumption is associated with increased risk of developing the MetS and its components such as elevated BP and hypertriacylglycerolaemia in middle-aged Korean women living in the urban area. Further studies are required to determine the effect of sex on the metabolic risks related to dietary factors and to enable the most appropriate dietary intervention by sex for the prevention and management of the MetS.

\section{Acknowledgements}

Epidemiological data used in this study were obtained from the KoGES (4851-302) of Korea Centers for Disease Control and Prevention, Republic of Korea.

This study was supported by the Basic Science Research Program of the National Research Foundation of Korea (NRF), funded by the Ministry of Education, Science and Technology (NRF2016R1D1A1B03931307). The NRF had no role in the study design, data analysis or writing of this article.

Y. K. helped with data acquisition, analysis and interpretation of the data and wrote the paper; J. K. contributed to the study design, analysis and interpretation of the data, wrote the paper and had primary responsibility for the final content. All the authors read and approved the final manuscript.

The authors declare that they have no competing interests.

\section{References}

1. Kit BK, Fakhouri TH, Park S, et al. (2013) Trends in sugar-sweetened beverage consumption among youth and adults in the United States: 1999-2010. Am J Clin Nutr 98, 180-188.

2. Vereecken CA, Inchley J, Subramanian SV, et al. (2005) The relative influence of individual and contextual socioeconomic status on consumption of fruit and soft drinks among adolescents in Europe. Eur J Public Health 15, 224-232.

3. Barquera S, Hernandez-Barrera L, Tolentino ML, et al. (2008) Energy intake from beverages is increasing among Mexican adolescents and adults. $J$ Nutr 138, 2454-2461.

4. Hu FB \& Malik VS (2010) Sugar-sweetened beverages and risk of obesity and type 2 diabetes: epidemiologic evidence. Physiol Behav 100, 47-54.

5. Barrio-Lopez MT, Martinez-Gonzalez MA, Fernandez-Montero A, et al. (2013) Prospective study of changes in sugar-sweetened beverage consumption and the incidence of the metabolic syndrome and its components: the SUN cohort. Br J Nutr 110, 1722-1731.

6. Chung S, Ha K, Lee HS, et al. (2015) Soft drink consumption is positively associated with metabolic syndrome risk factors only in Korean women: data from the 2007-2011 Korea National Health and Nutrition Examination Survey. Metabolism 64, 1477-1484.

7. Chan TF, Lin WT, Huang HL, et al. (2014) Consumption of sugar-sweetened beverages is associated with components of the metabolic syndrome in adolescents. Nutrients $\mathbf{6}$, 2088-2103.

8. Kim J, Jo I \& Joung H (2012) A rice-based traditional dietary pattern is associated with obesity in Korean adults. I Acad Nutr Diet 112, 246-253.

9. Lee H-S, Kwon S-O, Yon M, et al. (2014) Dietary total sugar intake of Koreans: based on the Korea National Health and Nutrition Examination Survey (KNHANES), 2008-2011. J Nutr Health 47, 268-276.

10. Ogden CL, Kit BK, Carroll MD, et al. (2011) Consumption of sugar drinks in the United States, 2005-2008. NCHS Data Brief 71, 1-8.

11. Kang Y \& Kim J (2016) Gender difference on the association between dietary patterns and metabolic syndrome in Korean population. Eur J Nutr 55, 2321-2330.

12. Ahn Y, Kwon E, Shim JE, et al. (2007) Validation and reproducibility of food frequency questionnaire for Korean Genome Epidemiologic Study. Eur J Clin Nutr 61, 1435-1441.

13. World Health Organization (1999) 1999 World Health Organization - International Society of Hypertension Guidelines for the Management of Hypertension. Guidelines SubCommittee. Blood Pressure Suppl 1, 9-43.

14. National Research Institute of Health (2011) Manual of Korean Genome and Epidemiology Study. Cheongju: Korea Centers for Disease Control and Prevention.

15. Alberti KG, Eckel RH, Grundy SM, et al. (2009) Harmonizing the metabolic syndrome: a joint interim statement of the International Diabetes Federation Task Force on Epidemiology and Prevention; National Heart, Lung, and Blood Institute; American Heart Association; World Heart Federation; International Atherosclerosis Society; and International Association for the Study of Obesity. Circulation 120, 1640-1645.

16. Hernan MA, Hernandez-Diaz S, Werler MM, et al. (2002) Causal knowledge as a prerequisite for confounding evaluation: an application to birth defects epidemiology. Am J Epidemiol 155, 176-184.

17. Schoenfeld D (1982) Partial residuals for the proportional hazards regression model. Biometrika 69, 239-241. 
18. Dhingra R, Sullivan L, Jacques PF, et al. (2007) Soft drink consumption and risk of developing cardiometabolic risk factors and the metabolic syndrome in middle-aged adults in the community. Circulation 116, 480-488.

19. Faeh D, Minehira K, Schwarz JM, et al. (2005) Effect of fructose overfeeding and fish oil administration on hepatic de novo lipogenesis and insulin sensitivity in healthy men. Diabetes 54, 1907-1913.

20. Jalal DI, Smits G, Johnson RJ, et al. (2010) Increased fructose associates with elevated blood pressure. J Am Soc Nephrol 21, 1543-1549.

21. Hwang IS, Ho H, Hoffman BB, et al. (1987) Fructose-induced insulin resistance and hypertension in rats. Hypertension $\mathbf{1 0}$, 512-516.

22. De Angelis K, Senador DD, Mostarda C, et al. (2012) Sympathetic overactivity precedes metabolic dysfunction in a fructose model of glucose intolerance in mice. Am J Physiol Regul Integr Comp Physiol 302, R950-R957.

23. Giacchetti G, Sechi LA, Griffin CA, et al. (2000) The tissue reninangiotensin system in rats with fructose-induced hypertension: overexpression of type 1 angiotensin II receptor in adipose tissue. J Hypertens 18, 695-702.

24. Duffey KJ \& Popkin BM (2006) Adults with healthier dietary patterns have healthier beverage patterns. J Nutr 136, 2901-2907.
25. Regitz-Zagrosek V, Lehmkuhl E \& Weickert MO (2006) Gender differences in the metabolic syndrome and their role for cardiovascular disease. Clin Res Cardiol 95, 136-147.

26. Reckelhoff JF (2005) Sex steroids, cardiovascular disease, and hypertension: unanswered questions and some speculations. Hypertension 45, 170-174.

27. Khalil RA (2005) Sex hormones as potential modulators of vascular function in hypertension. Hypertension $\mathbf{4 6}$, 249-254.

28. Nickenig G (2004) Should angiotensin II receptor blockers and statins be combined? Circulation 110, 1013-1020.

29. Knopp RH, Paramsothy P, Retzlaff BM, et al. (2005) Gender differences in lipoprotein metabolism and dietary response: basis in hormonal differences and implications for cardiovascular disease. Curr Atheroscler Rep 7, 472-479.

30. Li Z, Otvos JD, Lamon-Fava S, et al. (2003) Men and women differ in lipoprotein response to dietary saturated fat and cholesterol restriction. J Nutr 133, 3428-3433.

31. Tripathy JP, Thakur JS, Jeet G, et al. (2016) Urban rural differences in diet, physical activity and obesity in India: are we witnessing the great Indian equalisation? Results from a cross-sectional STEPS survey. BMC Public Health 16, 816 . 